\title{
Why Naunyn-Schmiedeberg's Archives of Pharmacology abandons traditional names of drug classes
}

\author{
Roland Seifert $^{1} \cdot$ Bastian Schirmer $^{1}$
}

(c) The Author(s) 2021, corrected publication 2021

Naunyn-Schmiedeberg's Archives of Pharmacology is the oldest existing pharmacological journal, celebrating its $150^{\text {th }}$ anniversary in 2023. In the journal, we place a lot of emphasis on good scientific traditions such as rigorous and fair peer review. We place science itself in the center and not its perceived "priority" or "impact". We also encourage discussion of weaknesses of studies and do not expect authors to submit "perfect stories".

However, traditions can also be deleterious if they do not adapt to the progress in scientific knowledge. And we do have such a case now and must adapt: Pharmacological nomenclature, particularly designations of drug classes, have continuously evolved over decades and are deeply rooted in pharmacological textbooks, the scientific literature, and, most importantly, in scientific language. Most names of drug classes are rather descriptive and often focus on clinical indications. Thus, drug class names rarely describe the precise mechanisms of action of drugs which, when the terms were coined, were mostly unknown. Moreover, historically, very little thought was given on consistence of drug class designations among subfields. For example, the prefix "anti_" is used for multiple drug classes acting against a certain disease, without specifying the mechanism of action. In addition, receptor antagonists are inconsistently labeled as "anti_," “_blockers,” or “_lytics.” Terminology was taken for granted. Three recent articles provide an overview of the problems associated with traditional drug class nomenclature (Seifert 2018; Seifert and Schirmer 2020, 2021).

Over time, an increasing mismatch between terminology and scientific facts evolved. Firstly, in many cases, we now better understand the mechanisms of action of drugs (Seifert 2019, 2021). Secondly, in many cases, drug uses have changed moderately or radically. For example, some

Roland Seifert

seifert.roland@mh-hannover.de

1 Institute of Pharmacology, Hannover Medical School, Carl-Neuberg-Str. 1, 30625 Hannover, Germany "antidepressants" are effective in polyneuropathy, and certain "antidiabetics" are effective in chronic heart failure and chronic kidney disease (Seifert 2019, 2021).

Based on these developments, 3 years ago, one of the authors of this Editorial proposed to critically rethink pharmacological nomenclature (Seifert 2018). Two years later, the authors of this Editorial proposed a simple nomenclature for psychoactive drugs in Naunyn-Schmiedeberg's Archives of Pharmacology (Seifert and Schirmer 2020) and earlier this year, we provided arguments why one of the most deeply rooted terms in pharmacology, i.e., "antibiotics," should be abandoned (Seifert and Schirmer 2020). The feedback from colleagues on these papers was very positive.

In parallel with the publication of the above-cited papers, a national process to restructure the medical curriculum was initiated in Germany, and the German medical community took the opportunity to abandon traditional drug class designations in the medical curriculum and to replace them with mechanistic or chemical terms (https://nklm.de/zend/ menu/index, May 24, 2021). This change in nomenclature is now being taught in all medical schools in Germany and will be the basis for the federal medical exams effective spring 2022 (https://www.impp.de/pruefungen/allgemein/ gegenstandskataloge.html, May 24, 2021). A textbook in German language fully adopting the nomenclature change has been recently published (Seifert 2021). An English version of the textbook, still just partially implementing the nomenclature changes, was published in 2019 (Seifert 2019). Because internationally, the textbook was very well received by professors and students for pharmacology curricula, a second edition of the textbook, fully implementing the nomenclature change, will now be prepared. Students enthusiastically embrace the new nomenclature as assessed by student evaluations because it is logical, avoids misunderstandings, and facilitates learning of pharmacology as well as the assignment of drug classes to new indications. Most importantly, from the perspective of students, a rational drug class nomenclature facilitates wording of fair and precise 
exam questions, specifically multiple-choice questions. From the physician-patient communication aspect, the new nomenclature is also useful because it avoids lengthy explanations, why, e.g., a patient should take an "antidepressant" for treating polyneuropathy.

At an online conference of the Nomenclature Committee of the International Union of Pharmacologists (IUPHAR) in April 2021, the nomenclature issue was presented and positively received as well. In addition, among editors of Naunyn-Schmiedeberg's Archives of Pharmacology, the nomenclature proposal received positive comments. It is agreed among authors and editors of Naunyn-Schmiedeberg's Archives of Pharmacology that literature searches with traditional terms such as "antidepressants," "antibiotics," "antipsychotics," and "non-steroidal anti-inflammatory drugs" become increasingly difficult and imprecise (Seifert 2018, Seifert and Schirmer 2020). It is also agreed among all stakeholders of Naunyn-Schmiedeberg's Archives of Pharmacology that changing an imprecise but long-established drug nomenclature in the scientific literature is difficult and cannot be accomplished immediately. Rather, it is a complex and continuous cultural process that takes time. However, doing nothing just because it is laborious is not an option in this case.

As with all cultural processes, the question is, when to start it? Should we wait, until IUPHAR has formally agreed on a new nomenclature? This process can take many years. Or should we start now as a kind of pilot project? It is a fact that at the level of teaching, pharmacology textbooks and exam questions, the process has already started, and the next generation of medical doctors and pharmacologists will be familiar with the new nomenclature from their training. But the next generation of medical doctors and pharmacologists will face the traditional nomenclature in the scientific literature and, of course, in many, if not most, internet resources. And established doctors and scientists educated in the old system might have communication problems.

Therefore, we feel that it is now high time to begin implementing the new pharmacological nomenclature not only in pharmacology textbooks but also in pharmacological journals. And this journal will make the first step. It was agreed with the Publisher of Naunyn-Schmiedeberg's Archives of Pharmacology, Springer Nature, that the nomenclature switch must not be abrupt but slow and smooth. This is important for allowing all involved parties, authors, editors, reviewers, and readers alike, a hopefully hick-up-free transition period.

Specifically, we require that authors of all future submissions to Naunyn-Schmiedeberg's Archives of Pharmacology principally use modern drug class nomenclature, either in the unabbreviated from or (preferably) abbreviated form. In general, readability of abbreviated drug class terms is better. In parentheses, authors are encouraged to use the traditional terms to avoid communication problems and ensure searchability of their papers in abstracting and indexing databases in the transition period. Most importantly, authors are not only required to implement the new nomenclature in the manuscript text but also in the abstract. Abstracts are globally and freely available to the scientific community in publication databases and not subject to subscription barriers. Thus, abstracts have great importance for global dissemination of knowledge, key concepts and terminology. It follows that use of modern drug nomenclature use will predominantly be propagated via abstracts. Manuscripts that do not abide to these new editorial guidelines will be administratively rejected and returned to authors for amendment.

We realize that initially, it will require some work from the side of authors to implement the required nomenclature changes, specifically since Naunyn-Schmiedeberg's Archives of Pharmacology is the first and currently only journal addressing this highly important topic. However, we are confident that our authors will soon appreciate that the precision and accuracy of their articles improve and that they can convey their scientific messages in a better and more precise manner.

The pilot project-type implementation of the nomenclature change in this journal is also important for IUPHAR and other pharmacological journals. In this way, these players will receive "real-world" feedback on which aspects of the nomenclature are good and practical and which aspects need revision.

Below, authors will find a table (Table 1) in which selected traditional drug terms are listed. In the future, for all future submissions to the journal, these terms must be avoided as primary terms in the abstract or manuscript text. Traditional terms should only be used in parentheses after the modern pharmacological terms (unabbreviated or preferably, abbreviated) have been introduced as primary terms. Table 1 also contains a number of problematic terms that should not be used in the future, mainly because they are ambiguous and not clearly defined. Rather, authors should specify the exact drug class they are referring to.

Authors should note that the list of traditional terms and translation into modern terminology and the list of problematic terms provided in Table 1 is not comprehensive. It just lists some of the most important traditional terms currently used in the literature. Many additional terms can be deduced from this table. Abbreviations for receptors, enzymes, transporters, and ion channels should follow IUPHAR rules (https://www.guidetopharmacology.org/nomenclature.jsp, May 24, 2021). For more details, authors are referred to previous publications on terminology of drug classes (Seifert 2018, 2019, 2021; Seifert and Schirmer 2020, 2021). A comprehensive discussion of many more problematic pharmacological terms will be the topic of a future publication to be submitted to this journal. 


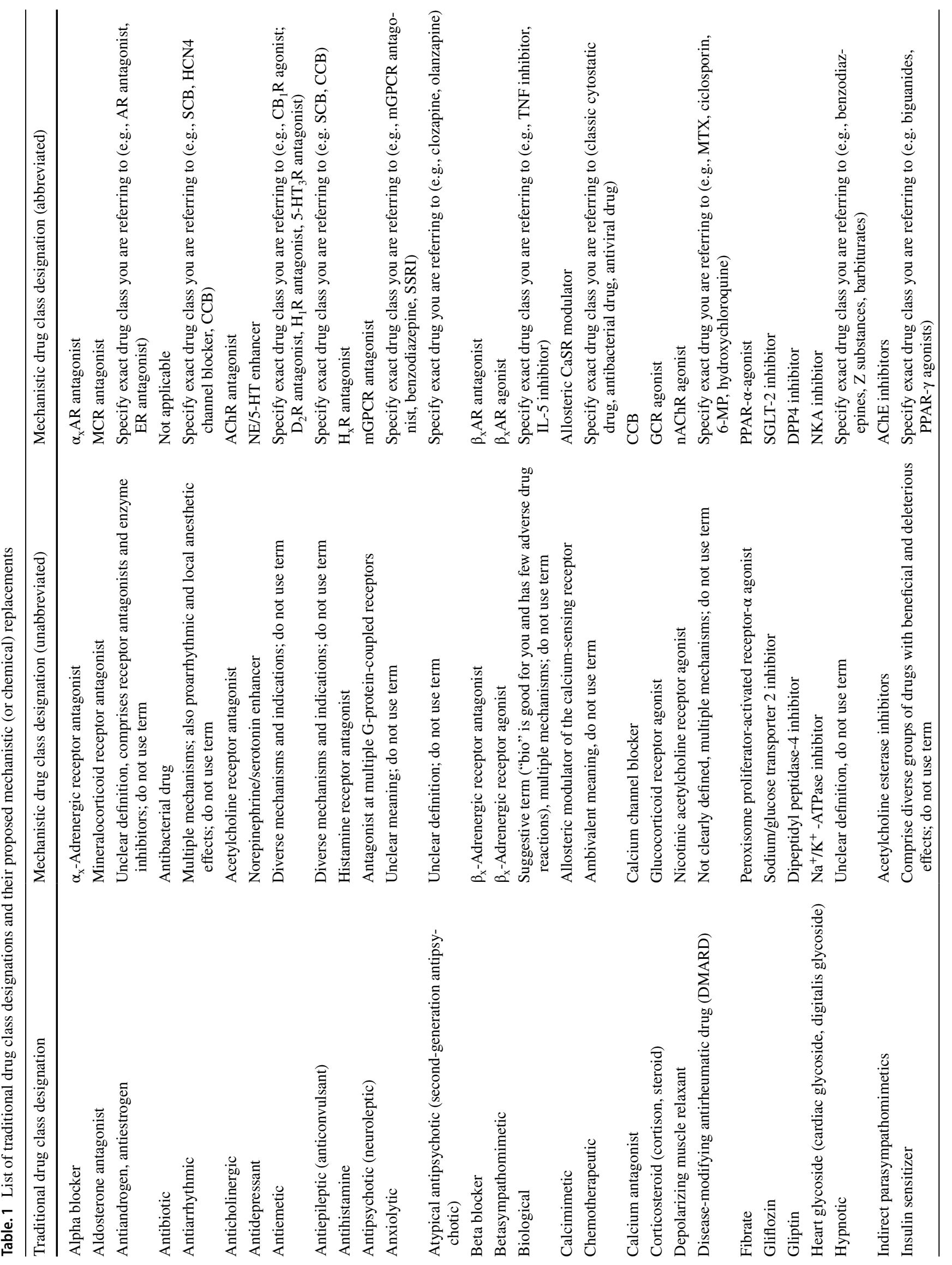




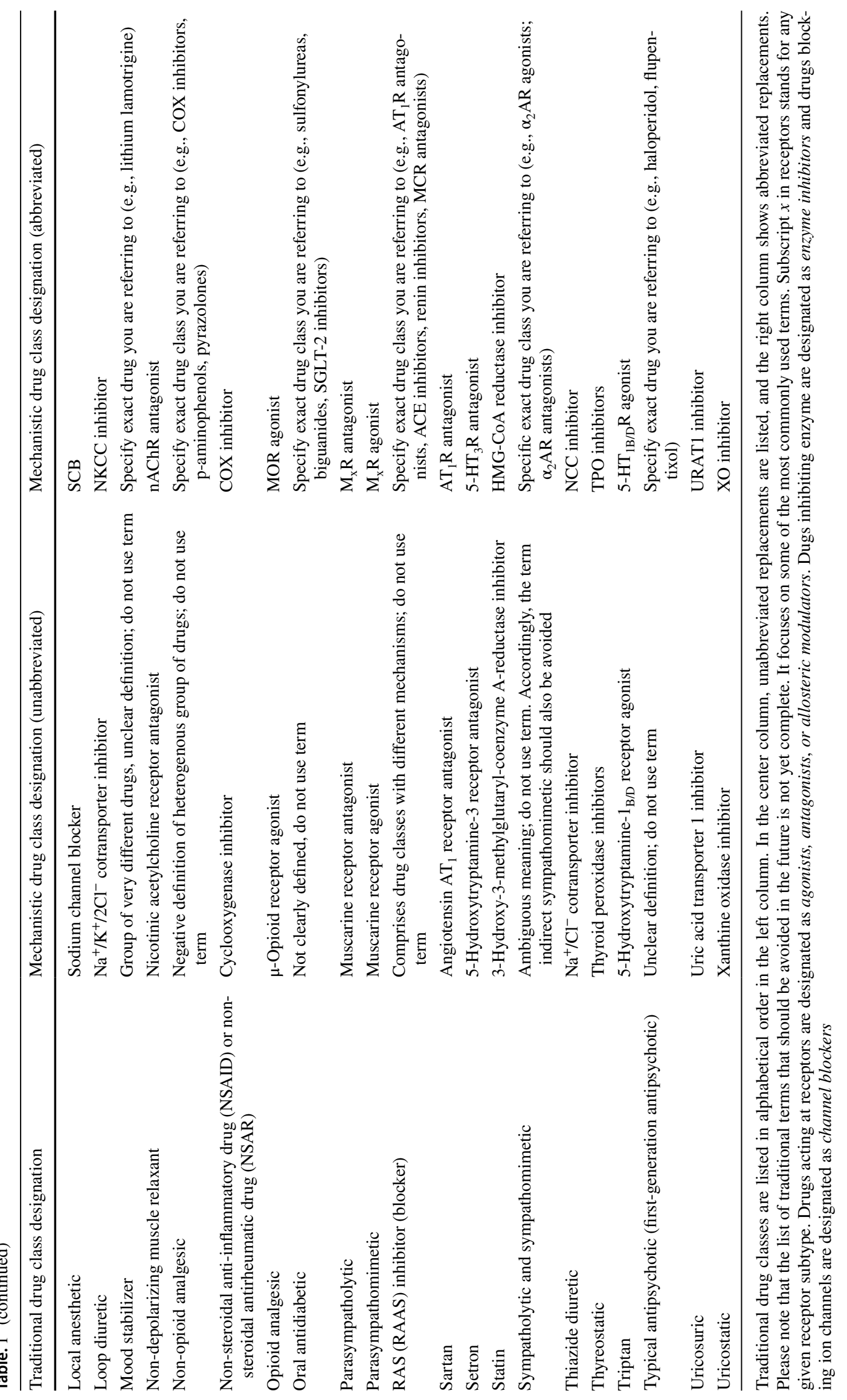


We encourage our authors and readers to provide feedback on the proposed nomenclature changes to the Editorin-Chief of Naunyn-Schmiedeberg's Archives of Pharmacology. Any suggestions for improvement of the nomenclature are most welcome. After all, it is a cultural process that must be accepted by the entire scientific and medical community to be effective.

To conclude, your papers will become cutting-edge both scientifically and with respect to modern terminology if you follow our editorial guidelines. And your critical pharmacological thinking will be sharpened as well. Please keep in mind that language shapes thinking and vice versa. And for pharmacology, both aspects should be as precise as possible.

Acknowledgements The authors acknowledge the stimulating discussions of the new drug nomenclature with the many colleagues from numerous medical disciplines, both nationally and internationally.

Funding Open Access funding enabled and organized by Projekt DEAL.

Open Access This article is licensed under a Creative Commons Attribution 4.0 International License, which permits use, sharing, adaptation, distribution and reproduction in any medium or format, as long as you give appropriate credit to the original author(s) and the source, provide a link to the Creative Commons licence, and indicate if changes were made. The images or other third party material in this article are included in the article's Creative Commons licence, unless indicated otherwise in a credit line to the material. If material is not included in the article's Creative Commons licence and your intended use is not permitted by statutory regulation or exceeds the permitted use, you will need to obtain permission directly from the copyright holder. To view a copy of this licence, visit http://creativecommons.org/licenses/by/4.0/.

\section{References}

Seifert R (2018) Rethinking pharmacological nomenclature. Trends Pharmacol Sci 39(9):785-797. https://doi.org/10.1016/j.tips.2018. 06.006

Seifert R (2019) Basic knowledge of pharmacology, Textbook. Springer, Heidelberg. ISBN 978-3-030-18899-3

Seifert R, Schirmer B (2020) A simple mechanistic terminology of psychoactive drugs: a proposal. Naunyn Schmiedebergs Arch Pharmacol 393(8):1331-1339. https://doi.org/10.1007/ s00210-020-01918-x

Seifert R (2021) Basiswissen Pharmakologie, Second Edition. Springer, Heidelberg. ISBN 978-3-662-60503-5

Seifert R, Schirmer B (2021) A case to stop the use of the term 'antibiotics'. Trends Microbiol. S0966-842X(21)00093-7. https://doi. org/10.1016/j.tim.2021.03.017

Publisher's note Springer Nature remains neutral with regard to jurisdictional claims in published maps and institutional affiliations. 